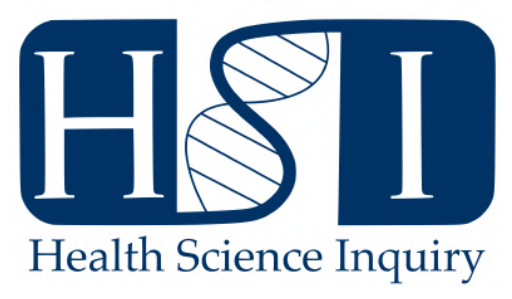

\title{
Routes Towards Treatment: Can We Cure HIV in the Future?
}

\author{
Farigol Hakem Zadeh ${ }^{1,2}$ \\ ${ }^{1}$ Department of Physiology, The University of Toronto \\ ${ }^{2}$ Ted Rogers Centre for Heart Research, Toronto
}

Since the first reported case of human immunodeficiency virus (HIV) infection in 1959, approximately 37 million individuals have been affected by this virus; this number is increasing by 1-2 million annually [1]. In HIV, mostly CD4+ T-cells, part of the human immune system, are invaded $[1,2]$. Several attempts to fight this epidemic have been successful in controlling the disease. For instance, with the immediate introduction of antiretroviral treatments after infection, the viral load can be controlled [3]. However, in most cases the virus re-emerges; thus, requiring lifelong therapy [3]. Amongst HIV-infected individuals, only two cases of complete cure through bone marrow transplantation from an HIV-resistant donor have been reported [4]. As such, novel approaches are being investigated with the aim of preventing, curing and completely eradicating the disease. Some of these potential treatments include "shock and kill", immune modulation, gene-editing techniques and stem cell transplantation.

In the "shock and kill" method, viral transcription and protein expression are activated from latency, resulting in the clearance of the virus through immune responses. Thus far, none of the clinical trials have shown a significant reduction in the number of virally reactivated cells [5]. The immune modulation method includes an overarching family of immunopotentiators (i.e. cytokines, immunostimulants), immunosuppressors (i.e. Trental), damagepreventing agents (i.e. antiviral agents) and immunization agents (i.e. active and passive immunization, vaccination) [6]. Currently, there are several trials attempting to generate HIV vaccines. However, this process is challenging due to the rapid mutation of HIV surface envelope proteins. Nonetheless, a recent study on the latest HIV vaccine, an active adenovirus-based immunization agent expressing the conserved region of the HIV envelope showed promising results [7]. This trial has recently moved to phase $2 \mathrm{~b}$. If all the safety and regulatory standards are met, this vaccine may protect humans against HIV in the future.

While HIV vaccine trials have been undertaken for more than three decades, alternative therapeutic approaches are also ongoing. Stem cell transplantation is another approach to fight HIV $[4,8]$. In this approach, the immune system of the infected individual is eliminated entirely and a new HIV-resistant donor immune system is substituted. Since the "Berlin Patient" in 2007, one more successful transplantation, the "London Patient" in 2019, has been reported
$[4,8]$. It is known that chemokine receptor type 5 (CCR5) and/or C-X-C chemokine receptor type 4 (CXCR4) are required for HIV virus entry into CD4+ T-cells. In CCR $5-\delta 32$ HIV-resistant individuals, the T-cells have the truncated version of CCR5 and therefore are resistant to HIV infection. However, one of the issues with relying on the HIVresistant cell transplantation approach is that there are few donors who have the natural mutations for HIV-resistance.

In the past, scientists generated CCR5 or the alternative CXCR4 co-receptor mutations through continuous expression of short-hairpin ribonucleic acids (shRNAs) [9]. Today, gene-editing techniques such as the zinc finger nucleases (ZFN), transcription activator-like effector nucleases (TALENs), and clustered regularly interspaced short palindromic repeats (CRISPR)/Cas9 are used to mutate CCR5 or CXCR4 co-receptors to block HIV infection. These methods can permanently obstruct receptor expression in hematopoietic stem/progenitor cells (HSPC) used for transplantation [10].

Some of the current clinical trials using ZFN are in phase 1. One of the most recent studies showed a safe infusion of ZFN-mediated CCR5-gene-modified autologous CD4+ T-cells in adults [11]. In this study, the decline of CCR5modified T-cells was significantly less than the unmodified T-cells, demonstrating HIV-resistance of these modified Tcells (ClinicalTrials.gov Identifier: NCT00842634). Other trials are currently looking at CCR5 disruption in HSPCs and possible engraftment of these modified cells in patients (Identifier: NCT02500849).

Because gene-editing is a new technique, investigating the future outcomes of HIV trials is procedurally and ethically essential to avoid concerns such as impaired immunity, preferred-trait selections and the generation of designer babies. Unfortunately, against ethical rules and regulations, CRISPR/Cas9 CCR5-modification was recently performed on human embryos by He Jiankui [12]. Jiankui claimed that through this procedure he generated the first geneticallyedited HIV-resistant human baby. This attempt led to criminal charges due to his disregard for the safety issues and the law. While the health of this baby needs to be monitored throughout the span of her lifetime, further restrictions are being implemented in research regulations to avoid any other future violations in gene-editing studies.

Overall, as we consider the implications of this controversial topic, the scientific community should recognize the 
great progress made in the field and the various promising routes that the new therapeutics hold to cure HIV. While antiretroviral therapy has revolutionized avenues for controlling HIV infection, several novel methods described have the potential to be combined in order to prevent and/or cure the disease. Therefore, all aspects of these methods, including their safety, efficacy and their ethical considerations, must be carefully assessed, refined and improved upon to ultimately lead us towards eradicating HIV.

\section{REFERENCES}

[1] Wang H, Naghavi M, Allen C, Barber RM, Bhutta ZA, Carter A, et al. Global, regional, and national life expectancy, all-cause mortality, and cause-specific mortality for 249 causes of death, 1980-2015: a systematic analysis for the Global Burden of Disease Study 2015. The lancet. 2016;388(10053):1459-1544.

[2] Sengupta S, Siliciano RF. Targeting the latent reservoir for HIV-1. Immunity. 2018;48(5):872-895.

[3] Luzuriaga K, Gay H, Ziemniak C, Sanborn KB, Somasundaran M, Rainwater-Lovett K, et al. Viremic relapse after HIV-1 remission in a perinatally infected child. New England Journal of Medicine. $2015 ; 372(8): 786-788$.

[4] Gupta RK, Abdul-Jawad S, McCoy LE, Mok HP, Peppa D, Salgado M, et al. HIV-1 remission following CCR $5 \Delta 32 / \Delta 32$ haematopoietic stem-cell transplantation. Nature. 2019;p. 1.

[5] Archin NM, Liberty A, Kashuba AD, Choudhary SK, Kuruc J, Crooks A, et al. Administration of vorinos- tat disrupts HIV-1 latency in patients on antiretroviral therapy. Nature. 2012;487(7408):482.

[6] Benson EM. Immune modulation in HIV infection: fact or fantasy? Journal of acquired immune deficiency syndromes. 1993;6:S61-7.

[7] Barouch DH, Tomaka FL, Wegmann F, Stieh DJ, Alter G, Robb ML, et al. Evaluation of a mosaic HIV-1 vaccine in a multicentre, randomised, doubleblind, placebo-controlled, phase $1 / 2 \mathrm{a}$ clinical trial (APPROACH) and in rhesus monkeys (NHP 13-19). The Lancet. 2018;392(10143):232-243.

[8] Cohen J. The emerging race to cure HIV infections. American Association for the Advancement of Science; 2011.

[9] Rossi JJ, June CH, Kohn DB. Genetic therapies against HIV. Nature biotechnology. 2007;25(12):1444.

[10] Allen A, Chung CH, Atkins A, Dampier W, Khalili K, Nonnemacher MR, et al. Gene editing of HIV-1 coreceptors to prevent and/or cure virus infection. Frontiers in microbiology. 2018;9:2940.

[11] Tebas P, Stein D, Tang WW, Frank I, Wang SQ, Lee $\mathrm{G}$, et al. Gene editing of CCR5 in autologous CD4 $\mathrm{T}$ cells of persons infected with HIV. New England Journal of Medicine. 2014;370(10):901-910.

[12] Cyranoski D. CRISPR-baby scientist fails to satisfy his critics. Nature. 2018;564(7734):13-14.

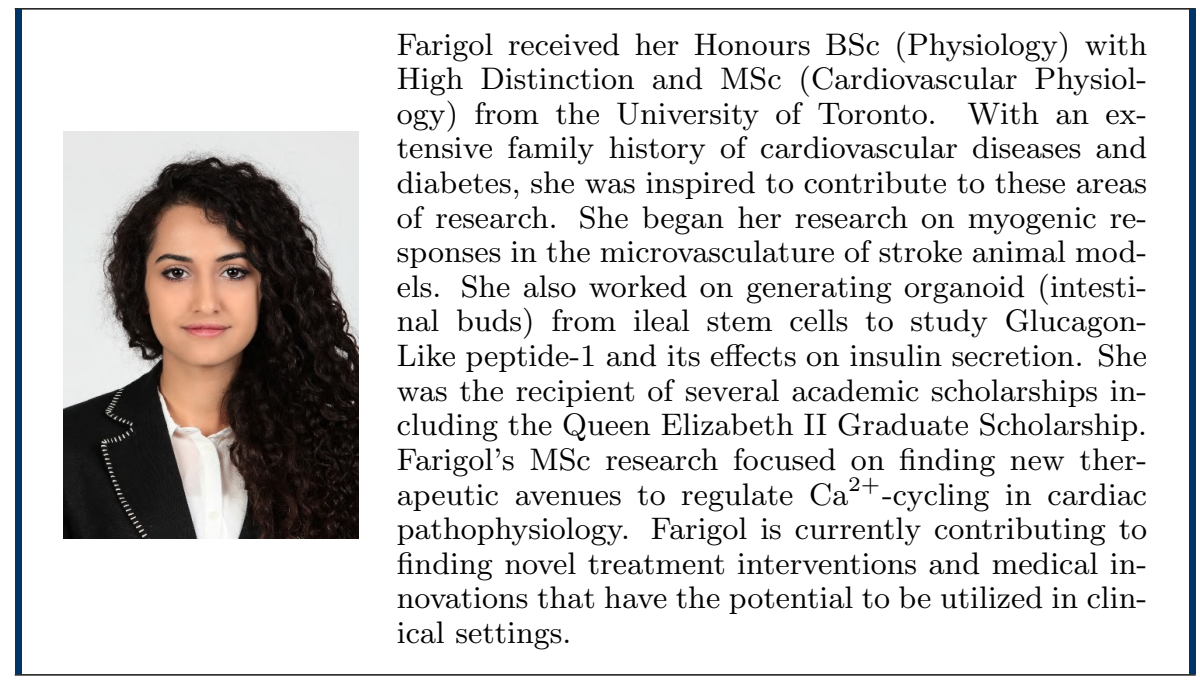

